\title{
A Mixed Reality Approach to Robotic Inspection of Remote Environments
}

\section{Document Version}

Accepted author manuscript

Link to publication record in Manchester Research Explorer

\section{Citation for published version (APA):}

Welburn, E., Wright, T., Marsh, C., Lim, S., Gupta, A., Crowther, B., \& Watson, S. (2019). A Mixed Reality Approach to Robotic Inspection of Remote Environments. Paper presented at UK-RAS Conference on 'Embedded Intelligence', Loughborough, United Kingdom.

\section{Citing this paper}

Please note that where the full-text provided on Manchester Research Explorer is the Author Accepted Manuscript or Proof version this may differ from the final Published version. If citing, it is advised that you check and use the publisher's definitive version.

\section{General rights}

Copyright and moral rights for the publications made accessible in the Research Explorer are retained by the authors and/or other copyright owners and it is a condition of accessing publications that users recognise and abide by the legal requirements associated with these rights.

\section{Takedown policy}

If you believe that this document breaches copyright please refer to the University of Manchester's Takedown Procedures [http://man.ac.uk/04Y6Bo] or contact uml.scholarlycommunications@manchester.ac.uk providing relevant details, so we can investigate your claim.

\section{OPEN ACCESS}




\title{
A Mixed Reality Approach to Robotic Inspection of Remote Environments
}

\author{
Elisabeth Welburn ${ }^{1}$, Thomas Wright ${ }^{2}$, Conor Marsh ${ }^{2}$, Sean Lim $^{1}$, Ananya Gupta ${ }^{1}$, William Crowther ${ }^{2}$ \\ and Simon Watson ${ }^{1}$
}

\begin{abstract}
Currently, inspection of remote environments poses potential hazards to the human operator. Mixed reality presents the unique opportunity for the human operators \& the piloted robots to co-exist within a virtual world that is then spatially mapped to the physical world. This can then act as a platform to conduct remote inspection, where the human operators could potentially possess heightened situational awareness and control.
\end{abstract}

\section{INTRODUCTION}

Remote inspection and asset management of offshore wind farms and their connection to shore is an industry that will be worth $£ 2$ billion annually by 2025 in the UK alone [1]. Deploying human personnel in such environments is often dangerous and costly. In these circumstances, robotic platforms displacing human personnel removes these associated risks. However, the viability of such an endeavor is contingent upon the robot's ability to maneuver effectively around the associated space. It is also imperative that the sensory data gathered is appropriate and sufficient for inspection of the infrastructure.

It is challenging to endow a robot with the capability to perform high-level mission planning based on environmental cues [2]. As a result, human involvement to varying degrees is often a necessity during robotic operation. Remote humanrobot interaction has been limited to a screen interface up until recent years; where there has been a shift to more involved mediums [3]. Here, the spatial awareness of the operator is limited by the sensing capabilities of the remote inspection robot.

Virtual reality (VR) is becoming increasingly more prevalent in human-robot interaction systems; an example of which is the construction of the surrounding three-dimensional space visualized in VR, that is based upon two-dimensional images taken by a camera mounted on a ground vehicle [4]. However, VR can be limited to interaction within the virtual world, resulting in a more passive role for the participant, such as walkthroughs and demonstrations.

Mixed reality (MR) environments are where both real and virtual world objects are presented together on a singular display [5]. Ideally, the mixed reality system is capable of realtime, bi-directional feedback between the virtual and physical worlds [6]. This concept is encompassed by the Digital Twin paradigm where a digital replica of a physical asset is constructed to aid real-time monitoring as well as mission forecasting. This could potentially boost safety and reliability through the creation of an immersive virtual environment that would be immediately reactive to any external disturbances [7].

MR presents the opportunity to use a virtual world to interact with real world physical environments, potentially increasing visibility of regions of interest. MR also presents a method to implement a simulated system alongside a realworld system. This could facilitate debugging by providing a direct comparison between simulated and physically deployed quadcopters during inspection.

This paper presents a novel Single Operator of a Singular Robot (SOSR) MR system that enables the operation of a physical quadcopter through the use of a digital twin that exists within a virtual world. This system forms the basis of a remote teleoperation system that will be developed within the future. It was developed as part of an outreach activity that was demonstrated at the Manchester Science and Industry Museum as part of the Manchester Science Festival 2018.

\section{LITERATURE REVIEW}

Perhaps the most engaging feature of MR is how it enables control of complex system via an intuitive interface. Rosen et al. sought to quantify this enhancement by directly comparing the use of a more traditional 2D display with MR. In this experiment, several participants were asked to decide whether a robot arm collided with certain obstacles using the two systems and no visualization mode. It was found that MR led to a reduction in task completion time by $7.4 \%$, increased precision by $11 \%$ and increased accuracy by $16 \%$ on average in comparison to the 2D display. The lack of a visualization interface was found to produce the worst results on average [8]

Human-robot interaction can take many forms; perhaps the most intuitive of which is the use of natural gestures. Crespo et al. sought to improve the quality of life of the elderly populace through the creation of a mixed reality interface to operate a drone. Interaction was enacted through the use of Rasberry $\mathrm{Pi}$ and additional 'Sense HAT' boards attached to the participants' arms. The data from the sensors was transmitted via Bluetooth to a smartphone that then ran the virtual reality simulation. This was subsequently visualized with Google cardboard headsets [9].

Honig et al. presented several use cases where mixed reality could be employed. The first of which was drones following humans in close proximity. The humans were visualized within the virtual world to ensure clearances were maintained. The

\footnotetext{
${ }^{1}$ School of Electrical and Electronic Engineering, University of Manchester.

${ }^{2}$ School of Mechanical, Aerospace, and Civil Engineering, University of Manchester.
} 
second was the creation of additional virtual agents to maximize area coverage of a physical, pre-existing robotic swarm. The third use case presented was the identification of Turtlebots using AR tags that are then read by a downwardfacing camera mounted on a virtual quadcopter flying overhead. Both virtual and physical Turtlebots were tracked using the simulated on-board camera. In both of these use cases, the physical robots deviated from the goal trajectories more due to latency and external disturbances, such as air currents [6].

Phan et al. used a mixed reality interface to allow for closed-loop control of multiple Crazyflie drones from multiple human operators within the same vicinity. The Unity client received current pose information from the VICON and PhaseSpace motion tracker systems and sent new goal positions to ROS base stations via a Python TCP socket connection. Commands are then transmitted to the Crazyflie drones with the use of a radio frequency radio. The path planning was implemented within Unity with the use of a navigation mesh generated by an $\mathrm{A}^{*}$ search algorithm. Markers were attached to obstacles, such as a physical door, as well as humans and drones. The markers are tracked and this data is utilized to inform rendering within the virtual world. Within the experiment, a pair of drones followed each human operator. As a human transversed through a door, the drones broke formation to follow suit. To ensure collision avoidance, a radius boundary of $0.5 \mathrm{~m}$ was placed around the rendered obstacles [10].

The enhanced spatial awareness MR endows the human operator in the study performed by Rosen et al. [8] is particularly beneficial when maintaining clearances in the remote inspection scenario considered in this paper. The use of natural, intuitive gestures to control the robot, as in Crespo et al. [9], reduces the perceived workload for the operator. As a result, a point-and-click mechanism was implemented to designate the waypoints for the quadcopter trajectory.

Both Honig et al. [6] and Phan et al. [10] investigated several use cases where drones were operated using MR in the vicinity of the human pilots. Within these studies, the drones were successfully navigated. This MR system was developed with a view of extending this concept for use in remote inspection operations.

\section{Methodology}

This section details the software architecture involved within the MR system. The interactions between different software components have been depicted in Figure 1.

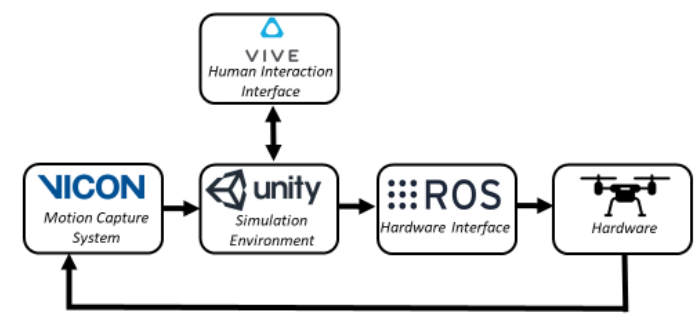

\section{A. Robotic Platform}

Remote environments are often difficult to access and navigate. In such situations, aerial robots enable superior coverage and flexibility during inspection. The selected morphology of the aircraft is a quadcopter due to their high maneuverability. A $27 \mathrm{~g}$ Crazyflie drone was selected as an exemplar robotic platform due to its small size, low price and Robot Operating System (ROS) compatibility.

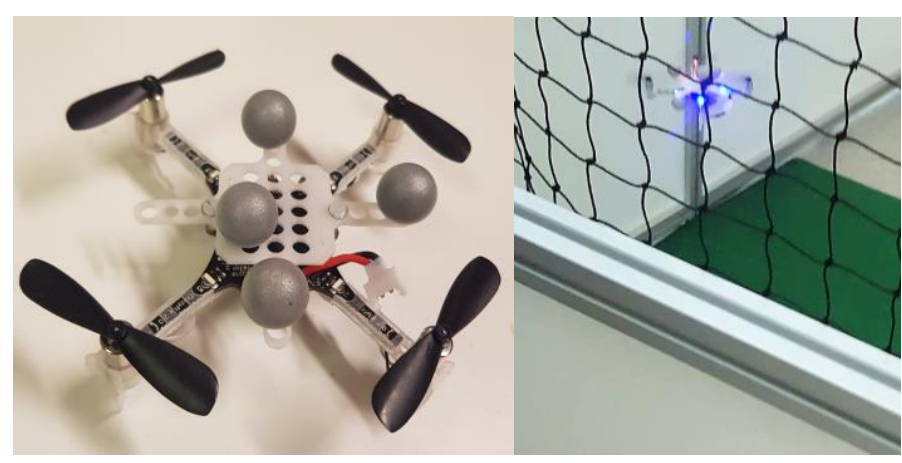

Figure 2: A Crazyflie Drone equipped with VICON markers.

\section{B. VICON Motion Capture System}

Markers were placed upon the Crazyflie drone as depicted in Figure 2. These were then selected and a rigid body object was created that represented the Crazyflie within the VICON space. The markers had to be placed asymmetrically in order to differentiate the axes of rotation of the Crazyflie. A ROS package, VICON bridge ${ }^{3}$, identified the DataStream server as a separate machine based upon the specified IP address.

The ROS node VICON bridge published the object pose data from the VICON in the form of a TransformStamped ROS message. A ROS node was written that subscribed to this and converted it into a PoseStamped ROS message that was then published.

The VICON system was set up using nine cameras attached to a steel rig in the demonstration, as depicted in Figure 3.

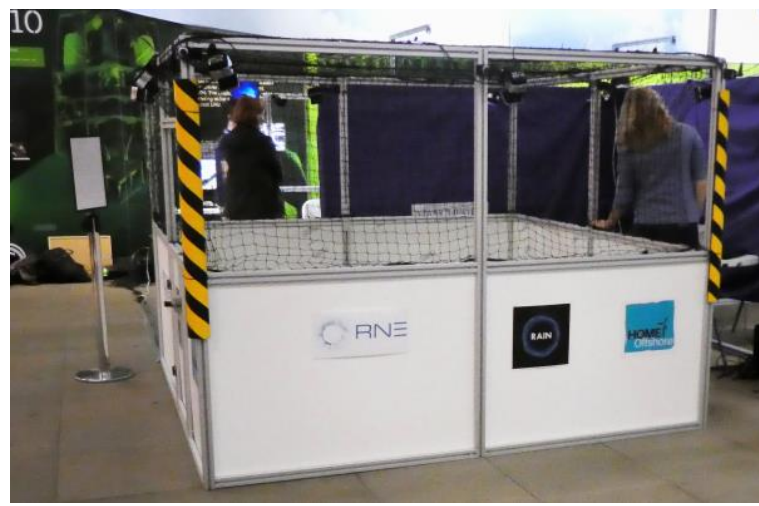

Figure 3: The VICON Cage to Capture the Crazyflie Movements at the Manchester Museum of Science and Industry.

Figure 1: Software Architecture of the Mixed Reality System. 


\section{Virtual Reality World}

The virtual reality system selected was the HTC Vive pro due to the pre-existing plugin, enabling compatibility with the game engine, Unity. The virtual world was constructed within Unity, utilizing the in-built physics engine. This consisted of a simulated quadcopter situated on an operational wind turbine. The operator was able to view the in-hand controllers within the simulation. A point-and-click mechanism was used to locate waypoints for the drone, where one waypoint exists at any one time. This method is intuitive for the human operator, reducing perceived workload. The current waypoint was represented by a red sphere in the virtual world. A red sphere was chosen because the stark contrast of the colour and morphology relative to the environment aids visualization of the waypoint within the 3D space. This was then published as the 'goal' position of the drone in the form of a PoseStamped ROS message.

An $A^{*}$ path planning algorithm was utilized within the Unity game engine. In order to enable a fast, on-the-fly remapping of the generated path, the target destination had to be relatively close of the current distance. This was ensured with the use of a Seeker component within Unity. This acted as an intermediate waypoint, accounting for the current trajectory and target destination. This mechanism had the effect of splitting the original trajectory into a series of segments. This Seeker component was published as a PoseStamped ROS message alongside the goal position.

\section{ROS Interface}

Both the goal and seeker component poses were visualized in Rviz alongside a Crazyflie URDF-generated robot model, animated using the VICON Bridge TransformStamped message. This was a useful debugging tool, and enabled fine tuning of the parameters of the seeker component within Unity.

The seeker component was published as the goal to the Crazyflie at a frequency of $120 \mathrm{~Hz}$. An on-board PID controller and IMU performed the low-level control during flight.

\section{Evaluations}

The Mixed Reality system outlined within this paper was demonstrated at the Museum of Science and Industry in Manchester, UK. It was observed that the Crazyflie drone successfully flew to the vicinity of the designated waypoint. A certain amount of overshoot was present within the trajectory. This may have been due to imperfect tuning of the PID values to account for the additional weight of the attached VICON markers. This could have also been affected by the constraint radius of the Seeker component being either too large or too small, not allowing for the application of sufficiently fast stabilizing torques.

The operators who, for the most part, had no experience in piloting drones, found the point-and-click mechanism intuitive to operate. However, some reported that the precise placement of the waypoints was difficult due to poor depth perception. This could be alleviated by offering an aerial view of the virtual space in conjunction to the current viewpoint based upon the operator's positioning.
Unfortunately, implementation of this would potentially increase the perceived workload of the human operator, reducing the sense of presence.

\section{CONCLUSIONS}

In conclusion, the mixed reality system outlined within this paper is applicable to tele-operation of a multitude of mobile robotic platforms in remote environments. The additional scaling and rendering functionalities coupled with the enhanced situational awareness for the human operator result in mixed reality being an invaluable tool for remote inspection.

Future planned work includes qualitative studies of the performance of human operators using MR systems to control remote inspection robots. Both static and dynamic obstacles could be inserted into the mixed reality to ascertain the navigational capabilities within confined areas. This SOSR system could also be further extended into a Single Operator of Multiple Robots (SOMR) system that could facilitate the coordination of a robot swarm. This system utilizes only the exocentric perspective of the controlled agent. In the future, this could be compared to an egocentric control interface in terms of spatial awareness and ease of use.

\section{ACKNOWLEDGMENTS}

The authors would like to thank the HOME-Offshore project (EPSRC reference: EP/P009743/1) and the Complex Autonomous Aircraft Systems Configuration, Analysis and Design Exploratory (CASCADE) project (EPSRC reference: EP/R009953/1) and the University of Manchester for the provided funding and support.

\section{REFERENCES}

E. M. Barnes et al., "Technology Drivers in Windfarm Asset Management Position Paper," pp. 1-46, 2018.

[2] J.-C. Latombe, Robot Motion Planning. Springer US, 1991

[3] T. Kot and P. Novák, "Application of virtual reality in teleoperation of the military mobile robotic system TAROS," Int. J. Adv. Robot. Syst., vol. 15, no. 1, Jan. 2018.

[4] L. A. Nguyen et al., "Virtual reality interfaces for visualization and control of remote vehicles," Auton. Robots, vol. 11, no. 1, pp. 59-68, 2001.

[5] P. Milgram and F. Kishino, "A Taxonomy of Mixed Reality Visual Displays," IEICE Trans. Inf. Syst., vol. E77-D, no. 12, pp. 1321-1329, Dec. 1992.

[6] W. Honig, C. Milanes, L. Scaria, T. Phan, M. Bolas, and N. Ayanian, "Mixed reality for robotics," IEEE Int. Conf. Intell. Robot. Syst., pp. 5382-5387, 2015.

[7] E. H. Glaessgen, D. T. Branch, D. S. Stargel, and M. Sciences, "The Digital Twin Paradigm for Future NASA and U . S . Air Force Vehicles," pp. 1-14, 2018.

[8] E. Rosen et al., "Communicating Robot Arm Motion Intent Through Mixed Reality Head-mounted Displays,” pp. 1-16, 2017.

[9] A. B. Crespo, G. G. Idrovo, N. Rodrigues, and A. Pereira, "A virtual reality UAV simulation with body area networks to promote the elders life quality," 2016 1st Int. Conf. Technol. Innov. Sport. Heal. Wellbeing, pp. 1-7, 2016.

[10] T. Phan, W. Honig, and N. Ayanian, "Mixed Reality Collaboration between Human-Agent Teams," 25th IEEE Conf. Virtual Real. 3D User Interfaces, VR 2018 - Proc., pp. 659-660, 2018. 